Síntesis:

El artículo expone la perspectiva que asume la Especialización en Comunicación Educativa, de Uniminuto, ante la investigación y la formación de investigadores en el campo de la Comunicación y Educación. En el primer subtítulo, Investigación en ciencias sociales: ¿Cómo conocemos la realidad social? se presentan los aspectos básicos de la investigación en ciencias sociales, sobre la realidad social, las dimensiones cualitativas y cuantitativas, la tarea del investigador, el abordaje y el proceso como tal; en el segundo, Investigación en Comunicación y Educación, se expone la configuración del campo comunicación y educación, de acuerdo con la visión de autores como Huergo, Martín Barbero, Soares, Espitia y Valderrama, para luego esbozar la investigación en este campo; se finaliza con Investigación en la Especialización en Comunicación Educativa (Uniminuto), en el que se argumenta la forma como se asumen los desafíos, desde este programa académico, para formar profesionales investigadores, quienes legan sus aportes a través de experiencias de investigación e innovación en el desarrollo de los trabajos de grado.

Palabras clave: investigación, Ciencias Sociales, Comunicación y Educación, formación en investigación Especialización en Comunicación Educativa.

Synthesis: The article presents the perspective that assumes the Specialization in Educational Communication of Uniminuto, with research and research training in the field of Communication and Education. In the first caption, social science research: How do we know the social reality? presents the basics of social science research on the social, qualitative and quantitative dimensions, the task of the researcher, the approach and the process as such, the second, Communication Research and Education, presents the configuration communication and education field, according to the vision of writers like Huerta, Martin Barbero, Soares, Espitia and Valderrama, and then outline the research in this field ends with Research in the Specialization in Educational Communication (Uniminuto) in argues that the way we take the challenges from the academic program to train professional researchers, who bequeathed his contributions through research experiences and innovation in the development of undergraduate work.

Key Words: Research, Social Sciences, Communication and Education, research training, specialization in Educational Communication.

\section{Formar investigadores en comunicación educativa, entre la teoría y la práctica}

\author{
Sandra Nieto Useche \\ Magíster en educación con Énfasis en \\ Docencia Universitaria, Especialista en \\ Comunicación-Educación,Técnica Profesional en \\ Diseño Gráfico y Comunicadora Social. \\ e-mail:sandranieto84@yahoo.com \\ Fecha de recepción: 01-11-2010 \\ Fecha de aprobación: 10-11-2010
}

\section{Investigación en ciencias sociales: ¿cómo conocemos la realidad social?}

\footnotetext{
Cuando surgen interrogantes acerca de un aspecto de la realidad, se indaga para lograr el conocimiento de las necesidades o de la situación problemática que se vive; entonces se inicia un proceso de investigación. El ser humano construyó órdenes culturales que se expresan en realidades sociales
} 
objetivas, que se mantienen en el tiempo debido a que se establecen unas pautas de comportamiento que hacen parte de ella. Los individuos asimilan aspectos objetivos y lo expresan en un nivel subjetivo?

La investigación comienza cuando el ser humano empieza a indagar acerca de los eventos que no puede explicar, comprender o aprehender. Aquí se encuentra ante una causa originaria o una situación problemática, que está al comienzo de la tarea investigativa, orientada a descubrir lo que no se conoce. Etimológicamente la palabra proviene del latín in (en) vestigare (hallar, inquirir, indagar); en su acepción más elemental el término se refiere a las acciones que llevan al individuo a "descubrir cosas"2.

En la realidad social se encuentran aspectos subjetivos y objetivos, las instituciones y el lenguaje median en la formación de la visión de mundo y en la comprensión de la vida social como una realidad objetiva. Así, el individuo asume las pautas de conducta de la cultura a la que pertenece, mediante la socialización y el 'ingreso' a las instituciones; las experimenta como inalterables, como una realidad objetiva no accesible a su memoria biográfica. El investigador se acerca a un grupo social y a un espacio específico; su comunicación, conocimiento previo, experiencia e interpretación determinan el inicio del proceso investigativo ${ }^{3}$.

El orden social depende de la institucionalización del comportamiento para que éste sea controlable; así, los miembros de una comunidad desempeñarán los

${ }^{1}$ Esta reflexión inicial se desarrolla a partir del texto Más allá del dilema de los métodos de BONILLA CASTRO, Elsy. RODRÍGUEZ SEHK, Penélope. Capítulo I. Bogotá: Grupo Editorial Norma. 2000, pág. 27.

${ }^{2}$ ANDER EGG, Ezequiel. Técnicas de la investigación social. Capítulo 3. Buenos Aires: Humanitas. 1983, pág. 57.

${ }^{3}$ BONILLA CASTRO, Elsy y RODRÍGUEZ SEHK, Op. cit. pág. 28. papeles que les asignen de acuerdo con los patrones de división de trabajo, parentesco y posición en el estrato social en el que se encuentren. A partir del rol se asume una ubicación en la sociedad y se esperan unos determinados comportamientos en las diversas interacciones que el sujeto tiene con los otros. Estos aspectos son esenciales para el investigador en sus recorridos iniciales, porque mediante ellos elabora los mapas espaciales y de relaciones interpersonales, que no siempre corresponden al orden jerárquico, y de esta manera reconoce los contextos.

El lenguaje es la capacidad inherente al ser humano que le permite comunicarse, hacer parte de la vida social, haciendo uso de diversos códigos; por eso se concibe como una forma de comunicación y de construcción del conocimiento, es un fenómeno social y constituye un conjunto de prácticas y acciones sociales; define las actividades en diferentes contextos y determina el proceso de legitimación de las instituciones. En la metodología cualitativa el investigador ve al escenario y a las personas desde una perspectiva holística, no son reducidos a variables, son considerados como un todo ${ }^{4}$. Entonces, desde este ángulo, los investigadores serán sensibles a los efectos que ellos causan sobre las personas que forman parte del contexto de ocurrencia en donde se desarrolla la investigación de su objeto de estudio. Son naturalistas porque interactúan con los informantes, de esta manera comprenden el escenario; cuando, por ejemplo, realizan las entrevista en profundidad acogen las dinámicas de una conversación normal e intentan controlar al mínimo los efectos sobre las personas con quienes interactúan en función de estudiar un determinado aspecto de la realidad. 
Bonilla y Rodríguez (2000) reconocen unos aspectos subjetivos de la realidad social, porque en las sociedades las personas se organizan en instituciones, actividades cooperativas y estructuras sociales que adquieren una estabilidad propia. La sociedad y las instituciones tienen unas características y unas funciones que parecieran existir independientemente de las personas.

Además, señalan que las diferentes teorías acerca de la exploración de por qué las personas se comportan de manera casi homogénea, conducen hacia la existencia de una intersubjetividad, realidades sociales compartidas, subyacentes, que explican cómo operan los aspectos objetivos de la realidad social. Por tanto, se construye la realidad institucional y se mantiene un orden social mediante la interacción o actividad comunicativa significativa entre las personas, que involucra un trabajo interpretativo mutuo. Así, los investigadores, desde una perspectiva cualitativa tratan de comprender a las personas dentro del marco de referencia de ellas mismas, es esencial experimentar la realidad tal como los otros la experimentan, para ello es necesario reconocer sus propias creencias, perspectivas y predisposiciones $^{5}$

Existen diferentes formas de explorar la realidad social, de conocerla y de reconocerla, los miembros de una comunidad tienen diversos tipos de conocimiento: el común y el científico. Como ya se expresó, cuando el ser humano comienza a indagar sobre las cosas inicia la investigación, se presenta una causa originaria o una situación problemática, que está al comienzo de la tarea

${ }^{5}$ Ibíd. pág. 29.

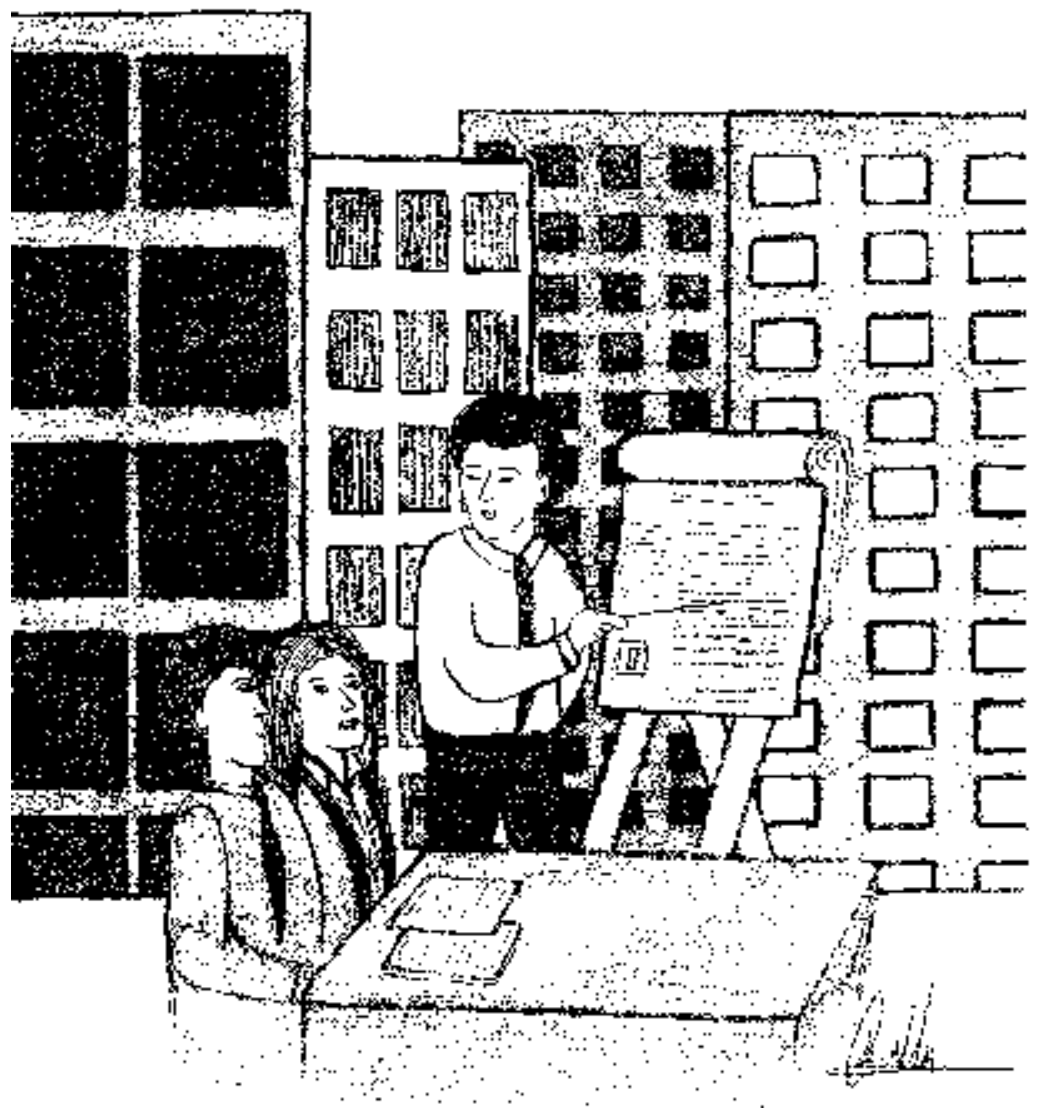

investigativa, orientada a descubrir lo que no se conoce. Conviene recalcar esta idea porque es aquí donde se ubica la investigación social, o el proceso que, a través del método científico, permite obtener nuevos conocimientos en el campo de la realidad social que estudia una situación para establecer necesidades y problemas con el fin de aplicar los conocimientos con fines prácticos ${ }^{6}$.

${ }^{6}$ Estos planteamientos tienen como referente el capítulo 3 de Técnicas de la investigación social de Ezequiel Ander Egg (1983), ya citado en este texto. 
En cuanto a los aspectos subjetivos, el ser humano busca explicaciones de esa realidad objetiva; es así como tanto la construcción de lo institucional y como la formulación del orden social se basan en la interacción, o la actividad en la que ellos se desenvuelven. En la vida cotidiana se establecen esas interacciones que hacen posibles diferentes niveles de conocimiento acerca de las acciones de los individuos.

Los niveles de conocimiento implícitos en el comportamiento de una sociedad configuran la dimensión cualitativa; desde aquí se obtiene un conocimiento directo de la vida social. Un estudio cualitativo es una forma de investigación sistemática desarrollada con procedimientos rigurosos no estandarizados. En la conceptualización del método científico de las Ciencias Sociales, se propone integrar los métodos cualitativos con los cuantitativos con la finalidad de enriquecer la comprensión de las realidades sociales; en consecuencia, si el investigador se sitúa sólo en uno de los métodos, limitaría las posibilidades del conocimiento del problema.

De ahí la importancia del abordaje de la investigación, el significado, el sentido, el diseño metodológico y la forma como se asume el trabajo de campo. Para Aracely de Tezanos, en el enfoque cualitativo interpretativo el trabajo de campo implica la inmersión del investigador en el fenómeno a estudiar. Permanecer en el territorio, convivir con las personas, a partir de sus percepciones construirá el objeto estudiado, esto distingue la modalidad del diseño?

${ }^{7}$ DE TEZANOS, Aracely. Una Etnografía de la Etnografía. Capítulo 2. EI abordaje de la indagación y Capítulo 3, Preguntas directrices. Bogotá: Antropos. 1998.
Cabe anotar que es necesario establecer la diferencia entre investigación y método. La investigación es una actividad de búsqueda, un conjunto de fases, de actuaciones sucesivas para hallar una respuesta a una situación que se ha presentado como problemática y el método son los procedimientos para seguir el camino de esa búsqueda, se expresa a través de normas y reglas genéricas de actuación científica ${ }^{8}$.

"El método científico predominante en las ciencias sociales es el hipotético-deductivo, los científicos prueban hipótesis a partir de un doble referente: el cuerpo conceptual y la realidad concreta que se estudia"9. "El método científico se aplica para fundamentar, justificar y respaldar hipótesis específicas"10 a partir de un marco conceptual. Sin embargo, en una investigación se corre el riesgo de darle énfasis a un seguimiento riguroso del método y sus reglas, porque el conjunto de reglas, normas y postulados es lo que institucionaliza la comunidad científica (Khun), así se jerarquiza y regula un proceso objetivo; pero en la búsqueda de la objetividad se pierde la formación integral y la capacidad de comprender e interpretar, por tanto el investigador debe reflexionar sobre la sociedad, sus dimensiones históricas y los distintos intereses de los grupos sociales, sin recaer en los extremos: exceso de rigor científico o predominio de la subjetividad.

Los marcos teóricos, de los cuales se deducen las hipótesis, parten de las revisiones de la literatura que en su mayoría corresponden a las tendencias conceptuales reconocidas por la comunidad científica de la cual hace parte, mientras que se descuida la pertinencia

\footnotetext{
${ }^{8}$ ANDER EGG, Ezequiel. Op. Cit. pág.59.

${ }^{9}$ Ibíd. pág. 41.

${ }^{10} \mathrm{Ibíd}$. pág. 42.
} 
de éstas para reflexionar de manera apropiada sobre el problema que se estudia. Lo teórico y lo metodológico son inseparables, entonces "se requiere que los supuestos que fundamentan el cuerpo conceptual del que se derivan las hipótesis deban ser explícitos"11, de estos depende "la percepción que logre el investigador del problema que indaga" porque así es posible formular "las preguntas y las respuestas que busca"12.

Es importante que en el proceso de investigación no se desarticule la relación entre la concepción del mundo del investigador, la teoría y el método que se usa; ni descuidar el dominio de su historia, de su cultura y de los conocimientos de los marcos conceptuales y metodológicos ${ }^{13}$.

La investigación cualitativa conceptualiza lo social como una realidad construida que se rige por leyes sociales, por una normatividad cultural ${ }^{14}$. La sociedad conforma una realidad objetiva que corresponde al andamiaje institucional, legal y normativo y sostiene un orden social específico que puede parecer inmutable; éstos corresponden a los aspectos formales; y una realidad subjetiva, o el modo como esa realidad es conocida, interpretada y expresada por los individuos que hacen parte de un marco institucional objetivo, corresponde al contenido de esa forma. Entonces lo objetivo hace referencia a los aspectos formales, mientras que lo subjetivo remite al contenido, de esa manera se conciben como dos dimensiones inseparables de la realidad ${ }^{15}$.

\footnotetext{
${ }^{11}$ Ibíd. pág. 43.

12 lbíd.

${ }^{13}$ Ibíd. pág. 44.

${ }^{14}$ MOONEY Y SINGER (1988), citado por BONILLA Y RODRÍGUEZ, pág. 69.

${ }^{15}$ LEFEBVRE (1982), citado por Bonilla y Rodríguez, pág. 69.
}

Si se establece una comparación entre las estrategias metodológicas es posible observar que lo cuantitativo analiza lo social buscando establecer cómo es la forma y lo cualitativo indaga por qué lo social toma esa forma. Mientras que, la investigación cualitativa se aproxima a las situaciones sociales para explorarlas, describirlas, y comprenderlas de manera inductiva. Parte de los conocimientos de las personas involucradas, de la interacción con otros miembros de su contexto social con quienes comparte significados y conocimientos de sí mismo y de la realidad ${ }^{16}$.

En consecuencia, el investigador llega al conocimiento cualitativo porque comprende el marco de referencia particular del grupo que estudia, define las etapas del proceso investigativo de acuerdo con la exploración de la situación que analiza. El acercamiento a la realidad y a los informantes depende de la relación personal que establece con ellos; así es posible que identifique los grupos, las situaciones, las interacciones y los informantes representativos de la realidad ${ }^{17}$. De esta manera, las etapas de la investigación cualitativa, es decir, la caracterización de la situación, el diseño metodológico, la recolección, la organización, el análisis y la interpretación de datos se retroalimentan y se confrontan de manera permanente, no se separan.

Entonces, se reconocen dos dimensiones la cualitativa y la cuantitativa que posibilitan el conocimiento; éste depende de la capacidad humana para percibirlo, explorarlo e interpretarlo. Por eso, desde las ciencias sociales, la investigación, desde un enfoque etnográfico, y un diseño cualitativo, se sustenta en la inmersión del investigador en el contexto de ocurrencia, en la

\footnotetext{
${ }^{16}$ Ibíd. pág. 70

17 Ibíd.
} 
observación, el reconocimiento, la participación del fenómeno que estudia.

Resulta esencial para la consolidación del conocimiento en las Ciencias Sociales, la habilidad, la experiencia y la capacidad fundada en la formación académica del investigador, puesto que son claves para garantizar la calidad del trabajo. En la construcción del conocimiento, el investigador combina la creatividad y la crítica en el uso de los métodos convencionales conforme a la situación que estudia. Bonilla y Rodríguez (2000) proponen los siguientes aspectos para superar las limitaciones de los métodos, con la finalidad de resolver lo que se plantea en el sugestivo título de su obra, Más allá del dilema de los métodos:

"Lograr una visión total del problema que estudia, tanto de manera cualitativa como cuantitativa.

Respetar la percepción y la interpretación que tengan los individuos y el grupo de la realidad.

Trabajar en equipo, así escucha, mira, capta lo observado para registrarlo y procesarlo de manera sistemática"18.

El investigador necesita de un conocimiento fundamentado para el desarrollo de su capacidad crítica; éste lo adquiere en su formación académica, mediante la cual adquiere experiencia. Es una persona y un profesional que enfrenta los aspectos de las técnicas cualitativas: flexibilidad, creatividad, sistematización, agudeza para entender las relaciones que existen entre fenómenos sociales.

En síntesis, el investigador es el eje central del proceso investigativo, mediante el cual desarrolla la capacidad de captar los rasgos esenciales que identifican la

${ }^{18} \mathrm{Ibíd}$. págs. 71-72. situación que estudia, de acuerdo con las percepciones de los sujetos que interactúan en ella. Para ello debe reconocer la diferencia entre sus opiniones y los argumentos válidos, pertinentes sobre la situación que investiga; por tanto se esfuerza por ser consciente de que sus valoraciones preconcebidas pueden distorsionar lo que capta, de todo esto dependerá el conocimiento, el registro, la selección, la interpretación de la información ${ }^{19}$.

\section{Investigación en comunicación y educación}

En primer lugar, tratar de ubicarse en el campo de la comunicación y educación resulta una tarea compleja, puesto que éste ha venido configurándose a través de un proceso histórico social en el que ha perfilado su objeto de estudio que, a la vez es intrincado y multifacético. Su complejidad radica en las distintas articulaciones que lo distinguen, es decir, el carácter vinculante entre comunicación, educación y transformaciones culturales ${ }^{20}$

Antes de avanzar, cabe dejar en claro que los autores se refieren al campo estableciendo la relación de varias formas: comunicación y educación; comunicación/ educación o comunicación-educación; la distinción entre una forma u otra no es meramente tipográfica, también y sobre todo, encierra el sentido en el que se definen y usan los términos. Huergo, por ejemplo, argumenta la relación comunicación/educación (a la manera de Schmucler, 1984), con los propósitos de recuperar los procesos de vinculación/expresión/liberación, aunque se pierdan los objetos disciplinares delimitados por un

${ }^{19}$ BONILLA y RODRÍGUEZ. Op cit., pág. 74

${ }^{20}$ HUERGO, Jorge. Comunicación, cultura y educación: una genealogía. La Plata: Universidad Nacional de la Plata. 2005. [Consultado: 05/09/2010]. Disponible en: http://jorgehuergo.blogspot.com/ 
afán cientificista, o a costa de la posible ruptura con los "imperialismos" de las disciplinas (cfr. Martín-Barbero, 1989); reconocer los contextos históricos, socioculturales y políticos (además de los campos disciplinares) donde surgen o se originan los problemas y las producciones teóricas (cfr. la perspectiva al respecto de Armand Mattelart); proponer algunas bases preliminares (que implican aquella recuperación y aquel reconocimiento histórico, y no una propuesta "fundacional") para la construcción de un espacio teórico transdisciplinario; es decir, devolver el carácter ético-político al campo de comunicación/educación. Desde esta perspectiva, aclara, el vínculo comunicación/educación también hace referencia a procesos y prácticas que se dan en los contextos de interrelación entre la cultura y la política. Así, el objeto de este campo es la articulación entre formación de sujetos y producción de sentidos ${ }^{21}$.

Ahora bien, en cuanto a los estudios culturales, la articulación se establece entre fuerzas sociales, que actúan en un momento determinado, que inciden en la formación del campo; pero, estas fuerzas no sólo convergen sino que establecen una relación en la que se reconoce el predominio o la posición que se asume. Además, la articulación implica que cada término, comunicación y educación, forman parte de un proceso de relación, en el que una vez iniciado cada uno se transforma ${ }^{22}$.

Jesús Martín Barbero aclara que, más que una cuestión de medios, el escenario de la comunicación debía ser una cuestión de fines para la educación, por eso la relación comunicación/educación es necesario

\footnotetext{
${ }^{21}$ Estos planteamientos pueden ampliarse en: HUERGO, Jorge (editor): Comunicación/Educación. Ámbitos, prácticas y perspectivas, Capítulo 1.(Puede verse, también, el sub-nodo "Comunicación/Educación", en el nodo VIAJES de la Revista Nodos de Comunicación/Educación, http://www.perio.unlp.edu.ar/nodos).

22 Ibíd.
}

ubicarla en el problema de fondo, que lo formula a través de dos interrogantes: "cómo insertar la escuela en un ecosistema comunicativo que es a la vez experiencia cultural, entorno informacional y espacio educacional difuso y descentrado. Y cómo seguir siendo en ese nuevo escenario el lugar donde el proceso de aprender guarde su encanto: a la vez rito de iniciación en los secretos del saber y desarrollo del rigor de pensar, del análisis y la crítica, sin que lo segundo implique renunciar al goce de crear" ${ }^{23}$.

Desde la perspectiva de Ismar Soares, en el año 2000 se sostenía que la educomunicación era un nuevo campo formado, que había adquirido autonomía, que se estaba consolidando. Una década después asevera que las prácticas educomunicativas, inicialmente alternativas, empiezan a movilizar grandes estructuras que buscan convertirse en programas de políticas públicas. Su incidencia se proyecta tanto en los grupos sociales como en la formación del sujeto, tal como lo expresa Huergo, quien enfatiza en la necesidad de avanzar en la autonomía de un campo que pueda instituir la palabra, una palabra que libere las representaciones, y manifieste un mundo que se apoye en un sueño común. Una autonomía que se sustenta en la política que reconoce a la sociedad autónoma, con hombres y mujeres autónomos ${ }^{24}$.

Agrega que la configuración de la relación comunicación-educación como campo no puede concebirse como espacio cerrado y predefinido. De esta manera es necesario asumir de forma

\footnotetext{
${ }^{23}$ MARTÍN-BARBERO, Jesús. Heredando el futuro. La educación desde la comunicación, En: Revista Nómadas. Bogotá: Universidad Central. 1997.

24 SOARES, Ismar. Caminos de la educomunicación: utopías, confrontaciones, reconocimientos. En: Revista Nómadas, № 30, abril. 2009.
} 


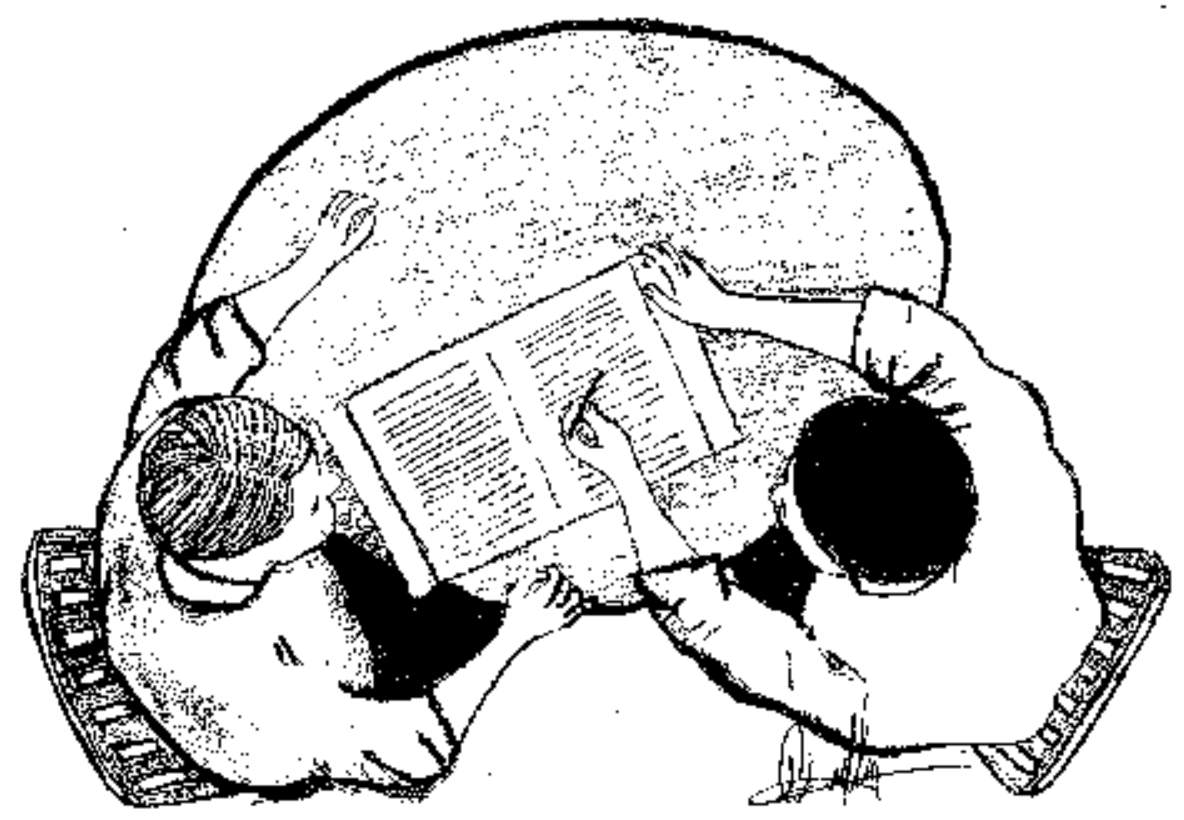

radical la búsqueda de una libertad especial, la libertad de la palabra. Por eso el autor sustenta que los educomunicadores distinguen como una utopía el valor estratégico de la lucha por la libertad de la palabra; esta lucha se concreta en acciones en los espacios educativos.

Soares reitera que el campo no se considera aislado en lo conceptual, en lo metodológico, ni en lo programático; lo sustenta su unidad conceptual movilizadora, aunque reconoce que es un concepto polisémico, que tiene diferentes formas del hacer. Cabe resaltar que coincide con Huergo cuando éste afirma que comunicación/educación es una política que asume la democracia como régimen del pensamiento colectivo, que libera la capacidad de "hacer pensante" y de la creatividad colectiva; es proyecto de autonomía y es posibilidad radical.
Espitia y Valderrama consideran que el campo ha venido configurándose a partir de cinco nodos de tensión, en los que confluyen tanto las trayectorias teóricas, como las trayectorias prácticas y profesionales. Es decir, los que atañen a la tensión entre el proyecto de escolarización de la modernidad y los proyectos emancipadores de las pedagogías críticas; al quiebre de las instituciones tradicionales como la familia, los partidos políticos, la iglesia y la escuela; otros se relacionan con el conocimiento mismo y con el lugar de la información, la comunicación, y el conocimiento en la sociedad contemporánea, que implican descentramientos en la educación y la comunicación y el quinto nodo hace referencia a la tensión inherente a la construcción de subjetividades ${ }^{25}$.

Por tanto, se reconoce como rasgo distintivo el movimiento permanente del campo; se trata de un campo relacional, que reconoce las constantes transformaciones culturales a las que se encuentra enfrentada la sociedad contemporánea. La invitación, desde sus orígenes, es recorrer un territorio a través de unas coordenadas topográficas de la comunicacióneducación trazadas por las trayectorias teóricas y las prácticas, tanto del proyecto moderno de escolarización como de los proyectos emancipadores. Algunos espacios que cruzan estas coordenadas son los

${ }^{25}$ ESPITIA VÁSQUEZ, Uriel. VALDERRAMA H., Carlos Eduardo. Hacia una apertura política del campo comunicación-educación. Revista Nómadas, No. 30l. Bogotá: Universidad Central. 2009. pág. 165. 
institucionales-educativos, los mediáticos-tecnológicos y los socio-comunitarios, entre otros ${ }^{26}$.

Situados en el plano de la investigación, iniciar un proceso de esta naturaleza implica abordar problemáticas en el campo de la Comunicación/Educación; sobre este particular Huergo expresaba hace casi una década que la investigación estaba en ciernes, pero no sucedía lo mismo con las preocupaciones prácticas que se han centrado en situaciones en torno al "uso de los medios y las nuevas tecnologías en el trabajo escolar, de la enseñanza a distancia, de proyectos de comunicación popular, de campañas educativas, etc. Esta preocupación práctica se ha plasmado en múltiples proyectos y producciones en Comunicación/Educación", ${ }^{27}$

También reconoce que esta investigación es más de tipo documental, que empírica; no obstante excluye a «las investigaciones sobre las "mediaciones múltiples" en los procesos de recepción televisiva, o las referidas al estudio sociocultural de las audiencias, o al análisis semiótico del currículum escolar y sus diferentes componentes ${ }^{28}$ ». A la vez, observa un cambio desde el campo de la comunicación, en el sentido de "la búsqueda de bases para investigaciones en comunicación/educación sobre la comunicación en el entramado de la cultura escolar, sobre la construcción de identidades y las nuevas formas de socialización (socialidad y nuevo sensorium), sobre la relación entre audiencias infanto-juveniles y educación, sobre mediaciones familiares y grupales, sobre discursos pedagógicos, etc." 29 .

\footnotetext{
${ }^{26}$ Ibíd., pág. 167.

${ }^{27}$ HUERGO, Jorge. Comunicación y Educación: aproximaciones. En: HUERGO, Jorge. Op. cit.

${ }^{28}$ lbíd.

${ }^{29}$ Ibíd.
}

\section{Investigación en la Especialización en Comunicación Educativa (UNIMINUTO)}

En el contexto de la Especialización en Comunicación Educativa, la Universidad enfrenta uno de los desafíos que surge del campo de la educomunicación, la formación de profesionales autónomos, íntegros, con la capacidad de gestar proyectos e incidir en las políticas públicas que pretenden la consolidación de una sociedad democrática, justa y que ofrece las posibilidades de participación ciudadana.

La misión de formar investigadores en este campo acoge la propuesta de identificar los esfuerzos realizados en la "lucha por la libertad de expresión por personas diferentes, en contextos diferentes, que se mantienen concentradas a prácticas semejantes, con el soporte de referentes teóricos y metodológicos próximos"30. Además, se reconoce la actuación profesional de los educomunicadores latinoamericanos, quienes "se caracterizan por su habilidad para coordinar proyectos culturales y facilitar la acción comunicativa de otras personas"; se preocupan por "la democratización del acceso a la información"; actúan en su práctica profesional como mediadores en la formación de valores solidarios y democráticos porque anhelan la transformación del ambiente en el que viven ${ }^{31}$.

El espacio académico de Trabajo de grado en la Especialización en Comunicación Educativa, es el propicio para identificar, sistematizar, desarrollar las experiencias de innovación e investigación que proponen los estudiantes durante su proceso de formación. Esta experiencia se desarrolla en tres fases. La primera, tiene como propósito orientar la presentación de una propuesta (como opción de

\footnotetext{
${ }^{30}$ Ibíd., pág. 196.
}

${ }^{31}$ Idem. 
grado), desde el campo de comunicación-educación dirigida a resolver un problema específico, acorde con los lineamientos institucionales, centrada en la producción de conocimiento desde la construcción de problemáticas de indagación y creación de estrategias que promuevan el cambio social.

La propuesta se considera una experiencia que debe estar inscrita en una de las modalidades: investigación, innovación, asistencia o co-investigación. Por ello constituye para los estudiantes, fundamentalmente, un ejercicio cuya finalidad es la de comprender un aspecto de la realidad, que los sitúa como agentes de transformación social y cultural y promotores de procesos de comunicación-educación en la construcción de una nueva ciudadanía.

Además se propone que los estudiantes de la Especialización identifiquen temas y problemas de investigación en el contexto de la Comunicación Educativa; y que construyan una propuesta de investigación en el contexto de los estudios sociales actuales, cuyo problema se ubique en el campo de comunicación y educación, y constituya un aporte a la línea del programa de Especialización y definan una propuesta metodológica clara y precisa, de tal manera que el proyecto sea un aporte relevante al campo.

Por tanto, otro de los desafíos que enfrenta la Especialización es el de "reinventar la educación", tal como lo plantea Jesús Martín-Barbero (2008), para ello identifica las consecuencias ocasionadas por las tranformaciones culturales en los modos en los que circula el saber; el des-centramiento del saber, un saber que ahora es disfuso, que es el descentramiento de la cultura occidental, cuyo eje era el libro, un saber que se fragmenta con la aparición del texto electrónico; des-localización/des-temporalización del saber, que se disemina, que supera las fronteras que lo separaban del saber común, que se escapa de los tiempos legitimados socialmente.

De esta manera, "en la investigación, la transdisciplina nombra los nuevos problemas de frontera que rebasan el saber especializado. Se trata de una encrucijada de agendas y de enfoques que está exigiendo superar la mera agregación de saberes y de resultados. Lo que se pone en juego es la capacidad de las instituciones académicas de rebasar, en la docencia como en la investigación, el plano de la renovación de contenidos o de técnicas, y afrontar el rediseño de los modos de producción del conocimiento, rediseño requerido hoy por una sociedad cuya compresión emborrona las fronteras de los saberes instituidos, al tiempo que desestabiliza las figuras profesionales establecidas" ${ }^{32}$.

En consecuencia se asume la investigación con carácter formativo, porque considera que un profesional del campo es "capaz de servir a la sociedad colombiana como un constructor de nuevos conceptos de la comunicación y la pedagogía para facilitar la convivencia en función de las necesidades sociales, que posibilite la calidad de vida y el bienestar individual y colectivo" ${ }^{\prime 3}$.

En conclusión, "Trabajo de grado, en sus momentos I, II y III, constituye un eje transversal al programa, en donde los estudiantes reciben orientación para la construcción y desarrollo de un proyecto de investigación. Como

${ }^{32}$ MARTÍN-BARBERO, Jesús. Saberes hoy: diseminaciones, competencias y transversalidades. Textos de la Cátedra de Comunicación y Educación. http://comeduc.blogspot.com/2008/07/jessmartn-barbero-saberes-hoy.html

33 UNIMINUTO, Facultad de Ciencias de la Comunicación. Proyecto Curricular del Programa Académico Especialización en Comunicación Educativa. Bogotá: Uniminuto. 2009 
tal constituye un proceso de estudio y una estrategia de articulación de temas que los diferentes ejes de profundización aportan en el programa. Así, la investigación que realice el estudiante debe brindarle la posibilidad de integrar sus conocimientos y dar soporte al perfil ${ }^{34}$ formativo del programa" ${ }^{\prime 35}$.

\section{El 'paso a paso' de la investigación}

Como ya se destacó, para el desarrollo de una propuesta de investigación es importante recordar que la realidad social tiene dos dimensiones inseparables: la cualtitativa y la cuantitativa; una estudia cómo es la forma y la otra por qué lo social toma esa forma. Para describir y comprender una situación se parte del conocimiento de las personas involucradas, de la interacción con otras personas en el contexto social y así, durante el proceso recopilar, interpretar, construir y compartir significados de esa realidad.

No se parte de certezas porque el objeto de estudio se construye en el proceso de investigación, lo que no conlleva una falta de rigurosidad, sino que a partir del conocimiento de la situación y de la retroalimentación de las etapas de la investigación se realiza la exploración; en ellas se interactúa con el grupo, con los actores participantes, que son representativos de esa realidad. Como investigador se deben tener en cuenta los siguientes aspectos: lograr la visión del problema con sus dimensiones cualitativas y cuantitativas; respetar la percepción e interpretación que tenga el grupo

34 "El especialista en Comunicación Educativa estará formado con capacidades de investigación para coadyuvar e intervenir con destrezas comunicativas, capacidad pedagógica, sensibilidad y espíritu crítico en las acciones comunicativas y pedagógicas en diferentes escenarios sociales, medios de información, instituciones educativas y organizaciones comunitarias". Ibíd, pág. 35.

${ }^{35}$ Ibíd. pág. 47.

\section{Aprendizajes}

de la realidad y trabajar en equipo. Además se deben identificar los rasgos esenciales de la situación y registrar e interpretar las percepciones del grupo acerca del problema.

Otro aspecto importante es la relación entre las preguntas directrices ${ }^{36}$ y las etapas del proceso de investigación, por ejemplo, a partir de la pregunta de investigación se pueden definir los objetivos: general y específicos. Y de acuerdo con los objetivos el nivel de la investigación, por ejemplo, descriptivo, clasificatorio o explicativo. Las etapas no se aíslan se encadenan para lograr un resultado.

Araceli de Tezanos expresa que el término "cualitativo" se usa indiscriminadamente para la perspectiva, el enfoque, el método, el diseño y que, por tanto, es necesario aclarar cada uno de estos conceptos, lo hace de manera sencilla: perspectiva, desde dónde se mira el problema: positivismo, fenomenológico, neoposivista, etc.; el enfoque, el punto que se focalizó del problema; el método, el modo de hacer ordenadamente algo. Por ello el enfoque es lo que denomina como cualitativo interpretativo.

Entonces, para retroalimentar esas etapas, en la planeación del proceso se pretende tener una visión de conjunto y de los pasos que involucra, es la bitácora de exploración que se debe seguir para conocer la realidad que se estudia.

Esta fase comprende un plan de trabajo referencial, que se formula a partir de la caracterización tentativa de las propiedades de la situación, con lo cual se perfila

\footnotetext{
${ }^{36}$ Araceli de Tezanos plantea que para cada etapa del diseño se formula una pregunta y las denomina "directrices", éstas deben quedar resueltas en el informe final.
} 
el trabajo de campo exploratorio en la primera etapa y de cuyos resultados se realizará la selección de la población que se observará, y así se escogerán las técnicas de recolección de información.

Además del proceso anterior, la etapa de recolección, la interpretación de la información, hasta la socialización de los resultados y el informe final, recogen el proceso que se desarrolla en las experiencias de investigación, y con algunas diferencias las de innovación que han propuesto los estudiantes de la Especialización. Entre los temas, que han sido objeto de reflexión, investigación e innovación , se encuentran los siguientes: educación ambiental en Bogotá; la investigación en formación ciudadana; la comunicación en el espacio escolar; construcción de ciudadanía desde la escuela; la escuela como espacio de encuentros y desencuentros; las prácticas comunicativas para la convivencia; la educomunicación; proyectos de innovación escolar y tecnologías de la información y de la comunicación; el periódico escolar; el periódico mural; periodismo en la radio escolar como herramienta educativa y pedagógica; implementación de medios escolares; la radio escolar como herramienta educomunicativa; la radio escolar como medio para la resolución de conflictos escolares; del texto al hipertexto; creación de blogs, revistas electrónicas, entre otros.

Una tarea que emerge al finalizar la primera década de este siglo, es la revisión sobre las investigaciones adelantadas tanto en América Latina, como en Colombia y en la ciudad-región en las universidades que tienen como misión formar especialistas, maestros y doctores el campo de la Comunicación/Educación, hecho que permitiría comprobar de qué manera se ha asumido el propósito expuesto por Huergo y que retoma Martín-Barbero: "ensanchar el territorio ubicando el campo de la comunicación-educación en la historia de las tradiciones pedagógicas latinoamericanas, tanto de las culturales y sus peculiares modelos de escolarización, como de las inscritas en las arquitecturas escolares con sus dispositivos de proxemia y de escenificación del poder, de disciplina y autoridad ${ }^{37 "}$.

En consecuencia para concretar este propósito es necesario afinar los procesos de investigación de diseño bibliográfico y compilar de manera crítica y contextualizada los frutos de la investigación causada en este terreno, acciones que dotarían de mayores posibilidades de fundamentación y de referentes aún más amplios, los procesos de investigación e innovación que se proponen en el contexto de la Especialización en Comunicación Educativa de la Universidad Minuto de Dios.

\footnotetext{
${ }^{37}$ MARTIN BARBERO, Jesús. Ensanchando Territorios Comunicación/ Cultura/Educación. En: Revista Nodos de Comunicación/Educación, $\mathrm{N}^{\circ}$ 1. La Plata, septiembre de 2002. http://cultura-mediosyeducacion. blogspot.com/2010/08/el-nuevo-texto-de-barbero-para-alumnos.html
} 


\section{Bibliografía}

ANDER EGG, Ezequiel. Técnicas de la investigación social. Capítulo 3. Buenos Aires: Hvmanitas. 1983.

BONILLA CASTRO, Elsy y RODRÍGUEZ SEHK, Penélope. Más allá del dilema de los métodos. Bogotá: Grupo Editorial Norma. 2000.

DE TEZANOS, Aracely. Una Etnografía de la Etnografía. Bogotá: Antropos. 1998.

ESPITIA VÁSQUEZ, Uriel y VALDERRAMA H., Carlos Eduardo. Hacia una apertura política del campo comunicacióneducación. Revista Nómada, No. 30. Bogotá: Universidad Central. 2009.

HUERGO, Jorge. Comunicación, cultura y educación: una genealogía. La Plata: Universidad Nacional de la Plata. 2005. [Consultado: 05/09/2010]. Disponible en: http://jorgehuergo.blogspot.com/

.Comunicación y Educación: aproximaciones. En: HUERGO, Jorge (editor): Comunicación/Educación. Ámbitos, prácticas y perspectivas, (Puede verse, también, el sub-nodo "Comunicación/Educación", en el nodo VIAJES de la Revista Nodos de Comunicación/Educación, http://www.perio.unlp.edu.ar/nodos).

MARTÍN-BARBERO, Jesús. Saberes hoy: diseminaciones, competencias y transversalidades. Textos de la Cátedra de Comunicación y Educación. http:// comeduc. blogspot.com/2008/07/jess-martn-barberosaberes-hoy.html

Ensanchando Territorios Comunicación/ Cultura/Educación. En: Revista Nodos de Comunicación/ Educación, Nº 1, La Plata, septiembre de 2002. http://

\section{Aprendizajes}

cultura-mediosyeducacion. blogspot.com/2010/08/elnuevo-texto-de-barbero-para-alumnos.html

,Heredando el futuro. La educación desde la comunicación. En: Revista Nómadas. Bogotá: Universidad Central. 1997.

SOARES, Ismar. Caminos de la educomunicación: utopías, confrontaciones, reconocimientos. En: Nómadas, No 30, abril. 2009

UNIMINUTO, Facultad de Ciencias de la Comunicación. Proyecto Curricular del Programa Académico Especialización en Comunicación Educativa. Bogotá: UNIMINUTO. 2009. 